\title{
The Significance of Transcapsular Blood Flow for Assessing Moderate to Severe Extrathyroidal Extension: Results of a Two-Center Study
}

\author{
Zhi Li $\mathbb{D}^{\prime}$, Shuqiang Chen $\mathbb{D}^{2}$, Jinguo $\mathrm{Li}^{3}$ \\ 'Health Management Center, The Second Affiliated Hospital of Fujian Traditional Chinese Medical University, Fuzhou, Fujian, People's Republic of \\ China; ${ }^{2}$ Ultrasound Department, The First Affiliated Hospital of Fujian Medical University, Fuzhou, Fujian, People's Republic of China; ${ }^{3}$ Ultrasound \\ Department, Fujian Medical University Union Hospital, Fuzhou, Fujian, People’s Republic of China
}

Correspondence: Jinguo Li, Email Lxy1990@I63.com

\begin{abstract}
Background: Extrathyroidal extension (ETE) is a crucial factor affecting the prognosis of thyroid cancer. Two-dimensional ultrasound is highly sensitive to ETE, but the sensitivity and specificity for severe ETE are unideal, so it does not improve the prognosis.

Materials and Methods: This retrospective study evaluated patients within three years of subcapsular thyroid cancer diagnosis with fine-needle aspiration (FNA). Routine ultrasound was performed and examined before surgery. In addition to the traditional twodimensional assessment method, particular attention was paid to transcapsular blood vessels, and the diagnostic efficacy of the two methods for mild, severe, and anterior and posterior ETE was compared.

Results: A total of 208 thyroid cancer nodules were included. Tumor size and gender were correlated with whether ETE occurred. The general method is more accurate for the overall identification of ETE but is not specific enough for severe ETE, while the transvascular method is the opposite. The two are similar in identifying the ETE of the anterior capsule, but the transvascular method has higher specificity in identifying the ETE of the posterior capsule.

Conclusion: The traditional two-dimensional method is highly sensitive to ETE detection in the anterior capsule, while transcapsular blood flow can more effectively detect extensive ETE and ETE in the posterior capsule.
\end{abstract}

Keywords: extrathyroidal extension, thyroid cancer, ultrasound, prognosis, metastasis

\section{Introduction}

Extrathyroidal extension (ETE) refers to the spread of tumors outside the thyroid into surrounding tissues. Approximately $30 \%$ of patients with differentiated thyroid cancer (DTC) are affected. ${ }^{1}$ The occurrence and range of ETE largely affect thyroid cancer staging as displayed in the American Association of Cancer TNM classification of thyroid cancer. Mild ETE typically invades the infrahyoid muscles of the neck, which is not deemed a negative predictor of survival or disease recurrence. American Thyroid Association (ATA) studies have shown that mild ETE has little effect on prognosis, while moderate to severe ETE do influence prognosis. ${ }^{2,3}$ Accurate preoperative evaluation of PTC'S ETE can help clinicians to select a reasonable and appropriate individualized and standardized treatment plan for PTC patients, reduce the postoperative recurrence rate of PTC, prolong patient survival and improve their quality of life. At present, the primary indices of ultrasonic evaluation of ETE include the contact degree between nodule and capsule (the contact length between nodule and capsule/the circumference of the nodule), the localized prolapse of thyroid capsule outline and echo interruption, etc., which are of certain value in predicting extrapedicular invasion. Existing ultrasound criteria are highly sensitive for diagnosing mild ETE that only involves infrahyoid muscles. However, the sensitivity to the highly invasive ETE or ETE with the posterior capsule involvement is lower than MRI. ${ }^{4}$ Studies have shown that the emergence of ETE is accompanied by neovascularization. Some scholars have even proposed that in the absence of ultrasound evidence of vascular structure or nerve infiltration, ETE ultrasound imaging cannot be considered a reliable indicator of actual 
extension. ${ }^{5}$ The issue remains that it is not clear whether moderate ETE and ETE penetrating the posterior thyroid capsule can be evaluated effectively by the sensitivity of blood flow signals. As such, the present study intends to investigate the relevance of this association.

\section{Methods}

This retrospective study was conducted at The Second Affiliated Hospital of Fujian Traditional Chinese Medical University and 1st Affiliated Hospital of Fujian Medical University from October 2017 to October 2020. It was approved by the Ethical Review Committee of the Second Hospital of Fujian Province and written informed consent was obtained from all participants. The study complies with the Declaration of Helsinki.

All patients underwent fine-needle aspiration (FNA) Cytology Biopsy and surgical operation. Routine standardized neck and thyroid ultrasonography were performed within three days prior to surgery. An ultrasound diagnostic apparatus (LOGIQ E9) and superficial variable frequency probe were used. The testing frequency was set to be 7 15 MHz. The two examiners had more than 10 years of experience in utilizing this technique. The nodules were located under the thyroid capsule and were in contact with the anterior and posterior thyroid capsule (no thyroid tissue was located between the nodules and the thyroid capsule). Ultrasound routinely identified the position, size, shape, boundary, halo, internal echo and calcification of nodules, peripheral blood vessels, and whether there were swollen lymph nodes on both sides of the neck.2.2 The patient was instructed to breathe calmly, and CDFI was activated at the same section under the condition of double image comparison. The size of the sampling frame was adjusted, the speed scale and gain were modified, and the image was stabilized. The clinicians then observed and recorded the relationship between nodules and precapsular thyroid, blood flow around nodules, and the relationship between blood flow and capsular.

Analysis of ETE was based on LEE's classification criteria (Exposure degree, thyroid capsule outline localized puffed, interrupted echo) and transcapsular blood vessels6. Transcapsular blood flow indicated that a single blood flow signal was observable between the nodule and the anterior thyroid capsule, and the blood flow extends across the capsule tissue outside of the thyroid. If the diagnostic results of the two evaluating doctors were inconsistent, the two doctors reassessed the image until agreement was reached and recorded. The pathology was followed up post-operation, and ETE was divided into gross and minimal according to the TNM standard7. Gross or extensive ETE (E-ETE) was defined as gross invasion of a tumor into the adjacent structures including adipose and skeletal muscles, sizeable vascular structures, and nerves. Minimal ETE (M-ETE) was defined as instances when thyroid cancer cells infiltrated the capsule but not including sizeable vascular structures, and nerves.

\section{Statistical Analysis}

We used Microsoft Excel to record original data and used SPSS 17.0 statistical analysis software for data analysis. Count data were expressed as a number (percent), and differences between groups were analyzed using the chi-square test. The measurement data were expressed as $\bar{x} \pm \mathrm{s}$. For variables that meet the normal distribution and homogeneity of variance, the independent sample $t$ test was used for data analysis. If the variable does not meet the normal distribution or the variance was uneven, the Kruskal-Wallis $H$-test was used. A P value $<0.05$ was considered statistically significant. For the two methods to evaluate ETE, E-ETE and M-ETE, chi-square and exact probability assessments were performed.

\section{Results}

\section{General Conditions of Thyroid Cancer Nodules}

A total of 208 nodules were included in the study, all of which were malignant nodules. The nodules were categorized from 199 cases of papillary carcinoma, 5 cases of medullary carcinoma, 4 cases of follicular carcinoma, and no undifferentiated type. General ultrasound information and ETE are described in Table 1. The result in Table 1 indicated that whether ETE appears is related to tumor size and gender. The E-ETE and M-ETE are related to size, gender, and location.

The boundary was blurred for the M-ETE in the anterior nodule of thyroid cancer on ultrasound, and no obvious transcapsular blood flow was seen (Figure 1). Postoperative examination showed that the tumor only invaded the 
Table I General Situation of Thyroid Cancer Nodules

\begin{tabular}{|c|c|c|c|c|c|c|c|}
\hline & \multicolumn{3}{|c|}{ ETE Group } & \multirow[b]{2}{*}{ Non-ETE } & \multirow[t]{2}{*}{$\mathbf{t}$} & \multirow[t]{2}{*}{$\kappa$} & \multirow[t]{2}{*}{$\mathbf{P}$} \\
\hline & G-ETE & M-ETETO & TOTAL & & & & \\
\hline Case number & 31 & 55 & 86 & 122 & & & \\
\hline Male & 19 & 20 & 39 & 29 & l & 3.56 & 0.03 \\
\hline Female & 12 & 35 & 47 & 93 & & & \\
\hline Age & $50.4 \pm 4.4$ & $47.6 \pm 4.1$ & $49.4 \pm 4.2$ & $47.6 \pm 3.5$ & 1.02 & I & 0.11 \\
\hline Maximum diameter line $(\mathrm{cm})$ & $2.2 \pm 0.43$ & $1.9 \pm 0.39$ & $2.2 \pm 0.4 I$ & $1.7 \pm 0.28$ & 2.98 & I & 0.04 \\
\hline Irregular edges & $23 / 8$ & $45 / 10$ & $68 / 18$ & $105 / 17$ & I & 0.56 & 0.09 \\
\hline Aspect ratio $>$ I & $26 / 5$ & $48 / 7$ & $74 / 12$ & $98 / 24$ & I & 0.76 & 0.08 \\
\hline Microcalcification (YES/NO) & $20 / 11$ & $4 I / 14$ & $61 / 25$ & $97 / 25$ & l & 0.94 & 0.06 \\
\hline Location (left/right) & $15 / 16$ & $29 / 26$ & $44 / 42$ & $64 / 58$ & l & 0.23 & 0.19 \\
\hline
\end{tabular}

Notes: Unless otherwise specified, the above data indicates the number of nodules (percentage); comparison between groups, $\mathrm{P}<0.05$ is statistically significant.

infrahyoid muscles.For posterior thyroid nodules, E-ETE, the ultrasound boundary was unclear, but transcapsular blood flow is visible (Figure 2).

The effectiveness of transcapsular blood flow method and general method for evaluating ETE is compared in Tables 2 and 3.

The sensitivity and specificity of the general method to the overall ETE were $87.2 \%$ and $82.3 \%$ respectively. There were no differences compared with the transcapsular blood flow method, which is $82.9 \%$ and $93.5 \%$ respectively. However, the sensitivity and specificity to E-ETE are $71.1 \%$ and $64.5 \%$, respectively.Using transcapsular blood flow as the criteria, the sensitivity and specificity were significantly improved to $90.3 \%$ and $90.9 \%$, respectively. Therefore, the general method was more accurate for overall recognition of ETE, but was not specific enough for severe ETE; meanwhile, the transvascular method exhibited the opposite evaluation.

Comparison of the effectiveness of the two methods to identify the ETE underneath the anterior and posterior capsule is reported in Table 4.

A comparison of the two methods reveals that they are equivalent in terms of identifying ETE status (mild and severe) of the anterior capsule, though the method of vascular invasion is more advantageous in finding the ETE of the posterior capsule, mainly due to its higher specificity.

\section{Discussion}

In patients with differentiated and medullary thyroid cancer, ETE is associated with reduced survival. In view of these findings, ETE should be included in the treatment guidelines for thyroid cancer. ${ }^{1}$ However, recent studies have shown

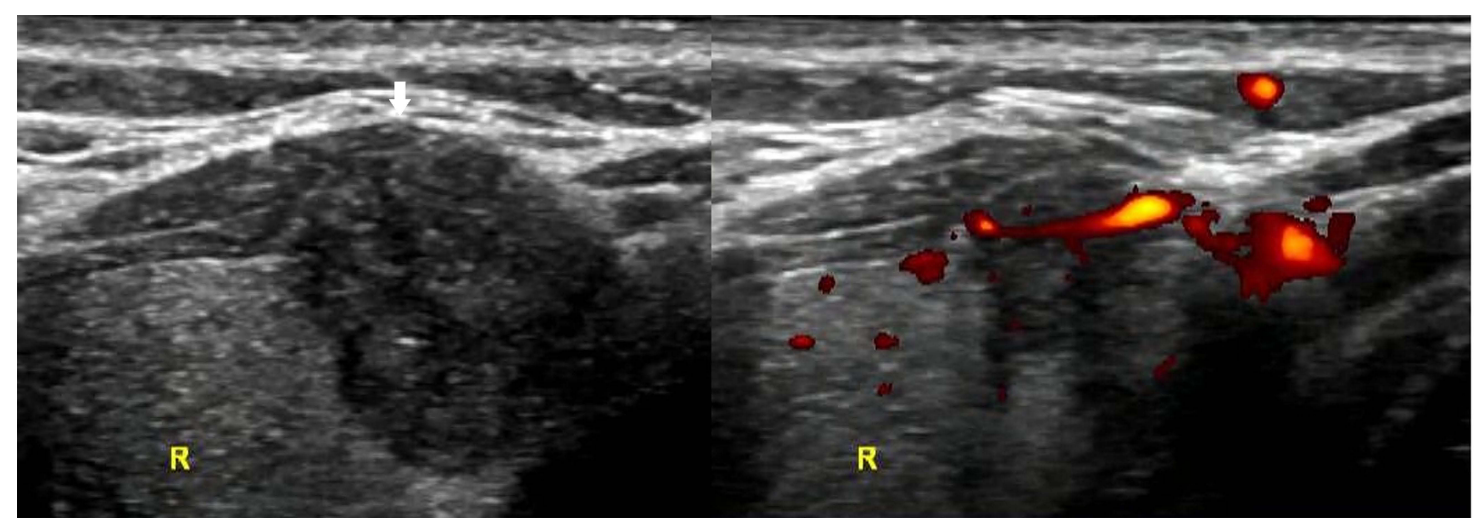

Figure I Thyroid papillary carcinoma locating in the anterior side is found blurry boundary with capsular, even extrusion feature in the gray US (arrow). But the missing of transcapsular blood flow lead to the judgment of min-ETE. The postoperative pathological results is displayed as invasion of cervical banded muscle. 


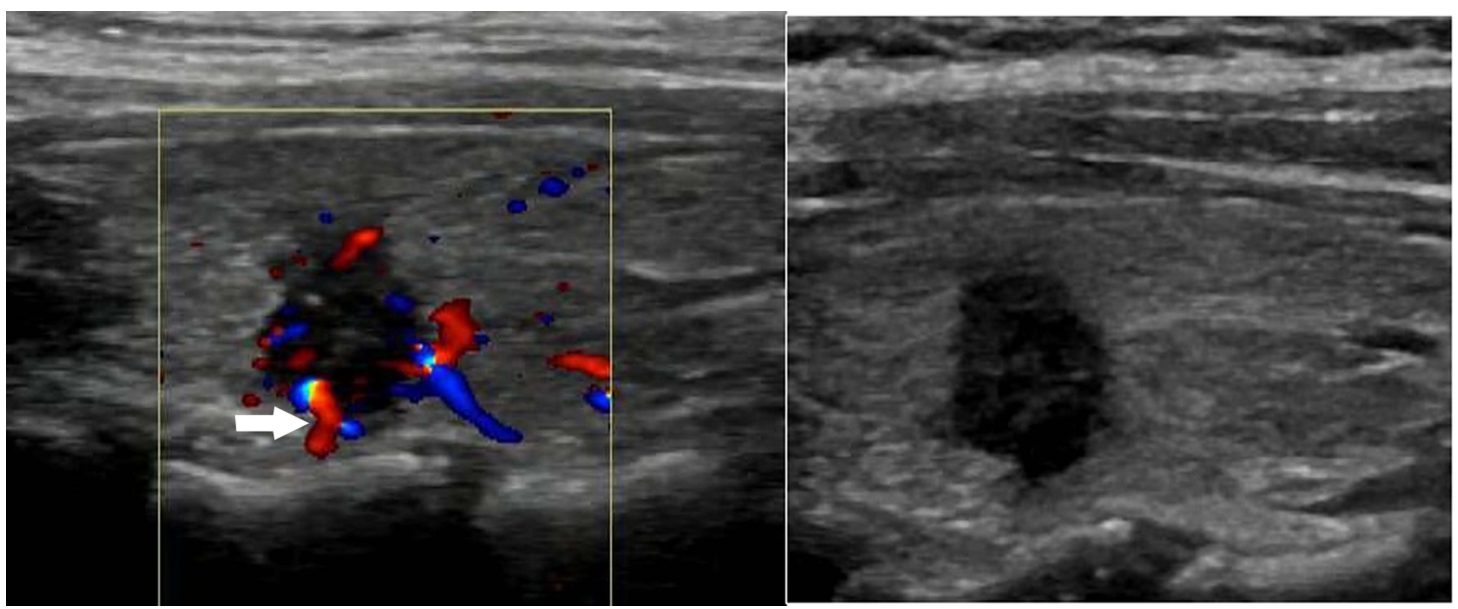

Figure 2 Thyroid papillary carcinoma locating in the posterior side, with clear boundary with capsular, is detected transcapsular blood flow. The postoperative pathological results is displayed as invasion of cartilagines tracheales.

that M-ETE has little effect on survival, while E-ETE is what affects central lymph nodes, distant metastasis, and longterm survival. ${ }^{6}$ The damage of E-ETE to local tissues greatly increases the difficulty of surgery. Therefore, E-ETE is particularly worthy of attention. Existing ultrasound evaluation of ETE signs mainly uses indicators such as the degree of contact between the nodule and capsule (the length of the contact between the nodule and the capsule/the circumference of the nodule), limited bulging of the thyroid capsule contour, and the interruption of echo. ${ }^{7}$ It has a certain value for predicting extracapsular invasion. But the above indicators mainly rely on the naked eye of sonographers, and it is difficult to achieve standardization and quantification. ${ }^{8}$ Using the existing evaluation system, it is difficult to determine the boundary between the thyroid tumor and the thyroid surgical capsule due to the protrusion of the capsule itself. This greatly complicates the determination whether the invasion occurs on a two-dimensional image. On the other hand, after the tumor invades the thyroid surgical capsule, the boundary becomes more blurred with the invasion of the cervical band muscle. It is difficult to determine whether only the banded muscle (ie M-ETE) is affected. Therefore, it is controversial to evaluate the ETE value, especially E-ETE, based on existing ultrasound methods. In this study, the sensitivity and

Table 2 Comparison of Efficacy of Two Methods for Evaluating ETE

\begin{tabular}{|l|c|l|l|}
\hline \multicolumn{2}{|c|}{} & E-ETE & M-ETE \\
\hline Location & Anterior & 71 & 54 \\
General method & Posterior & 51 & 32 \\
& - & 102 & 11 \\
Vascular invasion & + & 22 & 75 \\
method & - & 116 & 16 \\
\hline
\end{tabular}

Notes: -Means negative finding, + Means positive finding.

Table 3 Comparison of Efficacy of Two Methods for Evaluating Different Levels of ETE

\begin{tabular}{|l|c|l|l|}
\hline \multicolumn{2}{|l|}{} & E-ETE & M-ETE \\
\hline General method & - & 4 & 40 \\
Vascular invasion method & + & 27 & $1 \mathrm{I}$ \\
& - & 3 & 50 \\
\hline
\end{tabular}

Note: -Means negative finding, + Means positive finding. 
Table 4 The Efficacy of Two Methods to Evaluate the ETE Underneath the Anterior and Posterior Capsule

\begin{tabular}{|l|l|l|l|l|l|}
\hline \multirow{2}{*}{} & \multicolumn{3}{|l|}{ Anterior } & \multicolumn{2}{l|}{ Posterior } \\
\cline { 2 - 6 } & & + & - & + & - \\
\hline \multirow{2}{*}{ General } & + & 49 & 8 & 22 & 8 \\
& - & 5 & 66 & 10 & 43 \\
Vascular & + & 47 & 10 & 27 & 5 \\
invasion & - & 7 & 64 & 5 & 46 \\
\hline
\end{tabular}

Note: -Means negative finding, + Means positive finding.

specificity of evaluating ETE based on traditional standards are, respectively, $87.2 \%$ and $82.3 \%$, and the sensitivity and specificity for E-ETE are $71.1 \%$ and $64.5 \%$, respectively. Using the traditional standard approach promotes a high falsepositive rate. ETE especially, E-ETE, evaluation by two-dimensional signals alone, is not helpful in improving the prognosis of patients but may increase over-diagnosis and treatment.

The local infiltration and metastasis stages of malignant tumors involves breaking through the capsule, invading outwards, and then releasing local vascular growth factors. Neovascularization is a necessary condition for further extension and establishment of tumors9. Therefore, it is currently believed that detection of neovascularization will help to improve the detection of ETE10, especially E-ETE, and it has been suggested that, in the absence of sonographic evidence of infiltration of vascular structures or nerves, sonographic findings suggestive of ETE cannot be considered a reliable indicator of actual presence of ETE5. However, these conclusions are somewhat controversial. The present study found that the common method of ETE detection has high sensitivity and low specificity, while the new method is the opposite. Studies such as Zhang et al believe that it is the early invasion of the capsule, and the surrounding soft tissue may not cause echo11. This conclusion remains debatable, however. According to the general growth law of tumors, M-ETE should first cause disruption in the surrounding tissues of the thyroid gland. However, because it is difficult for two-dimensional ultrasound to identify fine boundaries, and if there is a fuzzy boundary, it is difficult to balance sensitivity and specificity. For E-ETE, the new method has better sensitivity and specificity. Gray-scale ultrasound can show subtle changes in cervical banded muscles, but it is difficult to distinguish the extent of ETE infiltration. The high sensitivity of the old method to ETE only exists in M-ETE, and there are a large number of false positives. For E-ETE, it seems non-effective. For patients with ETE, the effect of improving prognosis is limited. Therefore, the detection of E-ETE by vasculature is a more recommended method. Some scholars have also used Contrast Enhanced Ultrasound (CEUS) to find transcapsular blood flow to detect E-ETE11. Although the sensitivity is somewhat improved, in view of convenience and cost performance, we think it is enough to rely on convenient and easy color Doppler flow imaging (CDFI) or power Doppler imaging (PDI).

Due to the inherent characteristics of linear array probes, existing research on ETE is primarily focused on the anterior capsule. Due to the attenuation in the ultrasonic transmission (natural attenuation and significant attenuation of clustered calcified spots) and the complexity of deep structures, it seems difficult to find the ETE of the posterior capsule using only two-dimensional ultrasound. However, clinically, more E- ETE appears in the posterior capsule, and E-ETE in the posterior capsule is more likely to invade the surrounding tissues and increase the difficulty of the operation and lead to poor postoperative outcomes. In our study, the method of vascular discovery is more accurate than the traditional method for the posterior capsule. When the traditional method is used for evaluation, there are often hesitations due to the complexity of the surrounding tissue structure so incorrect decisions are made. The assessment of blood vessels can solve this problem adequately. This situation can be explained by the complexity of the vascular plexus posterior to the thyroid gland.

The present study also found that, as a whole, ETE is related to size and gender according to traditional methods. This agrees with the research of Lee YK and other scholars. ${ }^{9,10}$ Full resection is recommended for large nodules in males. For nodules larger than $4 \mathrm{~cm}$ or male thyroid nodules, the vessel difference between the anterior and posterior capsular has not been specifically compared due to the lack of sample size. 
We found that for the anterior capsular blood vessel, it is necessary to check from multiple angles, which is typical in a two-dimensional assessment approach. This is due to the fact that new blood vessels will not be exhibit such a regular morphology and arrangement.Additionally, it is advised to avoid pressurization especially for those blood vessels with a particularly tortuous shape, to avoid compressing neovasculature and causing false negatives. For blood flow of the posterior capsule, it is necessary to reduce the sampling frame and select the appropriate SCALE to obtain appropriate blood flow information in addition to the multi-angle inspection. Although detecting transcapsular blood flow is a powerful tool for detecting E-ETE and posterior capsular invasion, it is also necessary to combine the usual twodimensional indicators. Even the most accurate blood flow information is based on a good two-dimensional image. In the Thyroid Imaging-Reporting and Data System (TIRADS) classification standards proposed by ATA/ACR and various other institutions, little information is based on blood flow signals. ${ }^{11,12}$ In the foreseeable future, it is worth investigating further whether transcapsular blood flow signals are expected to be included in these standards.

The disadvantage of this study is that the number of samples is limited, especially due to the popularity of thyroid ultrasound examination, most nodules are relatively small, and nodules larger than $4 \mathrm{~cm}$ are rare. Therefore, the number of cases of E-ETE is relatively limited. As such, the E-ETE in males and posterior capsules requires further analysis.

The conclusion of this study: The traditional two-dimensional method is highly sensitive to the detection of ETE in the anterior capsule, and the transcapsular blood flow can more effectively detect E-ETE and ETE in the posterior capsule.

\section{Abbreviations}

ACR, American College of Radiology; ATA, American thyroid association; CDFI, Color Doppler flow imaging; CEUS, Contrast Enhanced Ultrasound; DTC, Differentiated thyroid carcinoma; ETE, Extrathyroidal Extension; E-ETE, Extensive ETE; FNA, Fine Needle Aspiration; M-ETE, Minimal ETE; PDI, Power Doppler imaging; TI-RADS, Thyroid Imaging-Reporting and Data System.

\section{Disclosure}

The authors report no conflicts of interest in this work.

\section{References}

1. Youngwirth LM, Adam MA, Scheri RP, Roman SA, Sosa JA. Extrathyroidal extension is associated with compromised survival in patients with thyroid cancer. Thyroid. 2017;27(5):626-631. doi:10.1089/thy.2016.0132

2. Zhang L, Liu J, Wang P, Xue S, Li J, Chen G. Impact of gross strap muscle invasion on outcome of differentiated thyroid cancer: systematic review and meta-analysis. Systematic review. Front Oncol. 2020;10:1687. doi:10.3389/fonc.2020.01687

3. Amit M, Boonsripitayanon M, Goepfert RP, et al. Extrathyroidal extension: does strap muscle invasion alone influence recurrence and survival in patients with differentiated thyroid cancer? Ann Surg Oncol. 2018;25(11):3380-3388. doi:10.1245/s10434-018-6563-x

4. Hu S, Zhang H, Sun Z, et al. Preoperative assessment of extrathyroidal extension of papillary thyroid carcinomas by ultrasound and magnetic resonance imaging: a comparative study. Comparative Study. Radiol Med. 2020;125(9):870-876. doi:10.1007/s11547-020-01184-0

5. Ramundo V, Di Gioia CRT, Falcone R, et al. Diagnostic performance of neck ultrasonography in the preoperative evaluation for extrathyroidal extension of suspicious thyroid nodules. Observational study. World J Surg. 2020;44(8):2669-2674. doi:10.1007/s00268-020-05482-6

6. Kuo EJ, Goffredo P, Sosa JA, Roman SA. Aggressive variants of papillary thyroid microcarcinoma are associated with extrathyroidal spread and lymph-node metastases: a population-level analysis. Comparative Study Research Support, N.I.H., Extramural. Thyroid. 2013;23(10):1305-1311. doi: $10.1089 /$ thy. 2012.0563

7. Kamaya A, Tahvildari AM, Patel BN, Willmann JK, Jeffrey RB, Desser TS. Sonographic detection of extracapsular extension in papillary thyroid cancer. J Ultrasound Med. 2015;34(12):2225-2230. doi:10.7863/ultra.15.02006

8. Grani G, Lamartina L, Cantisani V, Maranghi M, Lucia P, Durante C. Interobserver agreement of various thyroid imaging reporting and data systems. Endocr Connect. 2018;7(1):1-7. doi:10.1530/EC-17-0336

9. Lee YK, Kim D, Shin DY, et al. The prognosis of papillary thyroid cancer with initial distant metastasis is strongly associated with extensive extrathyroidal extension: a retrospective cohort study. Ann Surg Oncol. 2019;26(7):2200-2209. doi:10.1245/s10434-019-07314-x

10. Shin CH, Roh JL, Song DE, et al. Prognostic value of tumor size and minimal extrathyroidal extension in papillary thyroid carcinoma. Research Support, Non-U.S. Gov’t. Am J Surg. 2020;220(4):925-931. doi:10.1016/j.amjsurg.2020.02.020

11. Merhav G, Zolotov S, Mahagneh A, Malchin L, Mekel M, Beck-Razi N. Validation of TIRADS ACR risk assessment of thyroid nodules in comparison to the ATA guidelines. J Clin Imaging Sci. 2021;11:37. doi:10.25259/JCIS_99_2021 
12. Kim PH, Yoon HM, Hwang J, et al. Diagnostic performance of adult-based ATA and ACR-TIRADS ultrasound risk stratification systems in pediatric thyroid nodules: a systematic review and meta-analysis. Meta-Analysis Systematic Review. Eur Radiol. 2021;31(10):7450-7463. doi:10.1007/s00330-021-07908-8

\section{Publish your work in this journal}

The International Journal of General Medicine is an international, peer-reviewed open-access journal that focuses on general and internal medicine, pathogenesis, epidemiology, diagnosis, monitoring and treatment protocols. The journal is characterized by the rapid reporting of reviews, original research and clinical studies across all disease areas. The manuscript management system is completely online and includes a very quick and fair peer-review system, which is all easy to use. Visit http://www.dovepress.com/testimonials.php to read real quotes from published authors.

Submit your manuscript here: https://www.dovepress.com/international-journal-of-general-medicine-journal 